\title{
A PROPOSED MATHEMATICAL MODEL FOR THE VELOCITY PROFILE INTERNALLY TO A CONICAL DIFFUSER
}

\author{
D. L. M. Barbosa ${ }^{a}$, \\ D. A. T. D. R. Vaz ${ }^{a}$, \\ J. R. P. Vaz ${ }^{a}$, \\ S. W. O. Figueiredo ${ }^{a}$, \\ M. O. da Silva ${ }^{a}$, \\ C. J. C. Blancob, \\ and A. L. A. Mesquita ${ }^{a}$ \\ ${ }^{\mathrm{a}}$ Universidade Federal do Pará \\ Faculdade de Engenharia Mecânica \\ Instituto de Tecnologia \\ Campus do Guamá \\ Belém, Pará, Brasil - CEP 66075-110 \\ delimamb@gmail.com \\ ${ }^{\text {b} U n i v e r s i d a d e ~ F e d e r a l ~ d o ~ P a r a ́ ~}$ \\ Instituto de Tecnologia \\ Campus do Guamá \\ Belém, Pará, Brasil- CEP 66075-110 \\ Received: October 25, 2013 \\ Revised: November 21, 2013 \\ Accepted: December 30, 2013
}

Faculdade de Engenharia Sanitária e Ambiental

\section{ABSTRACT}

The use of diffusers around of the horizontal-axis wind turbines have been widely studied, since the diffuser provides an improvement in the turbine power coefficient. These diffusers are often called Diffuser Augmented Wind Turbines (DAWT's). The DAWT's have the feature to make efficiency exceeding the Betz limit (maximum energy flow extracted $=$ $59.26 \%$ ), due to the increasing of the internal mass flow by influence of the diffuser presence. Thus, the present work proposed a mathematical model describing the behavior of the velocity profile internally to a diffuser according to the characteristics of flow and geometry of a conical diffuser. The model results were compared with experimental data and showed good agreement.

Keywords: DAWTs, Diffuser, Renewable energy.

\section{NOMENCLATURE}

a distance from the origin to the input of the diffuser, $m$

$\mathrm{b}$, radius of the vortex, $\mathrm{m}$

c distance between the rings of the vortex, $m$

d inlet diameter of diffuser, $m$

D outlet diameter of diffuser, $m$

DAWTs Diffuser Augmented Wind Turbines

$d \vec{l} \quad$ elemental length of the vortex ring, $\mathrm{m}$

$d \vec{u}_{*} \quad$ elemental velocity field induced, $\mathrm{m} / \mathrm{s}$

$\mathrm{K}$ circulation, $\mathrm{m}^{2} / \mathrm{s}$

$\mathrm{L} \quad$ length of the diffuser

$\vec{r} \quad$ position vector, $m$

$r \quad$ radius of the diffuser, $\mathrm{m}$;

u velocity, $\mathrm{m} / \mathrm{s}$

$\mathrm{u}_{*} \quad$ velocity induced, $\mathrm{m} / \mathrm{s}$

$\mathrm{V}_{\mathrm{T}} \quad$ tangential velocity, $\mathrm{m} / \mathrm{s}$

$\mathrm{V}_{0} \quad$ undisturbed velocity, $\mathrm{m} / \mathrm{s}$

$\mathrm{r}, \mathrm{X} \quad$ system coordinates, $\mathrm{m}$

\section{Greek symbols}

$\phi \quad$ opening angle of the diffuser, rad

$\xi \quad$ proportionality constant to act as a correction factor in the Eqs. 3 and 4.

\section{Subscripts \\ 1 diffuser entry \\ 2 diffuser exit}

\section{INTRODUCTION}

The use of diffusers around of the horizontalaxis wind turbines aims at increasing the mass flow through the rotor. This effect provides a considerable improvement in the efficiency of the turbine, which extracts more energy of the flow, when compared with a turbine without diffuser (free flow turbines). A diffuser is a device which causes a pressure drop in the output region (suction region downstream of the diffuser). The pressure drop provides acceleration on 
the fluid particles within the diffuser, increasing the flow velocity near the entrance (Bussell, 1999). Oman et al. (1975), Foreman and Gilbert (1979) showed that increasing the velocity ratio between the plane of the rotor and undisturbed flow velocity can be two or more times, resulting in a proportional increase in the power coefficient of the turbine, exceeding the Betz (1926) limit, which is $59.26 \%$ in the case of the turbines without diffuser. Hansen et al. (2000) conducted a study on turbines with diffusers using Computational Fluid Dynamic (CFD), which the increasing of the velocity in the rotor plane was 1.83 for a case in which the geometry of the diffuser used was the NACA 0015 profile deformed. The main limitation of the horizontal-axis wind turbines design with diffusers is not considering a formulation to describe satisfactorily the influence of the diffuser geometry on the internal velocity profile. Thus, this paper analysed a mathematical model which describes the velocity profile of the internal conical diffuser, using the Biot-Savart law to calculate the velocity induced by a vortex ring. The results of the model were compared with experimental data.

\section{MATHEMATICAL MODEL}

In this paper, it has been described a mathematical model for the velocity profile internally to a conical diffuser, aiming its use in the efficient design of the horizontal-axis wind turbines. Therefore, to assess the velocity field in the diffuser, the flow was considered to be an overlap between the uniform flow and a flow caused by movement produced by a vortex ring. Thus, the movement can be defined as the amount of rotation of the fluid acting on the spreader due to the flow in which is immersed. The movement modifies the velocity and pressure fields around the diffuser, resulting in a resultant force, which is accompanied by a vortex ring, which mathematical model is established by the Biot-Savart law, resulting in an increased velocity within the diffuser.

$$
d \vec{u}_{*}=\frac{K}{4 \pi r^{3}} d \vec{l} \times \vec{r}
$$

where $d \vec{u}_{*}$ is the velocity field induced, $d \vec{l}$ is an elemental length of the vortex ring, $\vec{r}$ is the position vector and $K$ is the circulation (Eq. 1).

Figure 1 shows two rings vortices using the Biot-Savart formulation, in cylindrical coordinates, where it is necessary to define the vortex element and position vector in relation to the axis of symmetry.

The mathematical model considers the following assumptions: conical diffuser, the suction outlet of the diffuser is modeled using a vortex ring; the lock due to the presence of the diffuser on the flow is modeled considering a vortex ring invert steady and unidirectional. Therefore, applying the
Biot-Savart law and taking only the velocity in the direction along the axis of the diffuser, there is the velocity induced by vortex rings.

$$
u_{*}(x)=\frac{1}{2}\left\{\frac{K_{2} r_{2}^{2}}{\left[r_{2}^{2}+\left(L+a-x+c_{2}\right)^{2}\right]^{\frac{3}{2}}}-\frac{K_{1} r_{1}^{2}}{\left[r_{1}^{2}+\left(x-a+c_{1}\right)^{2}\right]^{\frac{3}{2}}}\right\}
$$

where, as shown in Fig. 1, $r_{2}$ is the radius of the diffuser outlet, $r_{1}$ is the radius of the diffuser inlet, $L$ is the length of the diffuser, $a$ is the distance from the origin to the input of the diffuser, $c_{1}$ e $c_{2}$ are the distances between the rings of the vortex inlet and outlet of the diffuser, respectively (Eq. 2). For calculation of the circulations $K_{1}$ e $K_{2}$, it was considered the geometry of the diffuser, the behavior of the flow around the diffuser and the structure of the vortex formed in the entrance and exit of the diffuser as shown in Fig. 2.

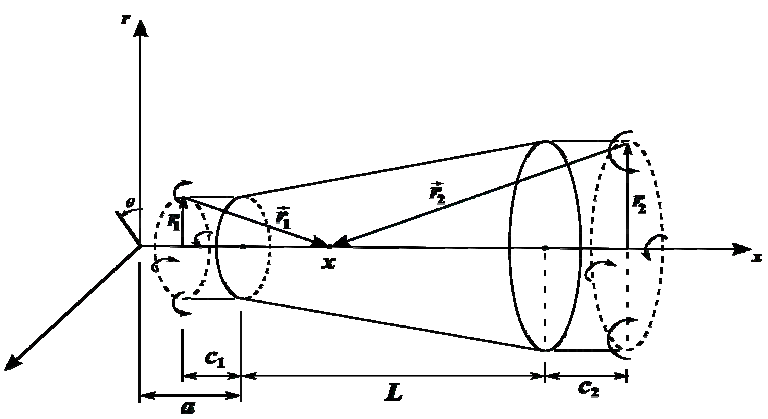

Figure 1. Representation of the geometry model and the vortex rings.

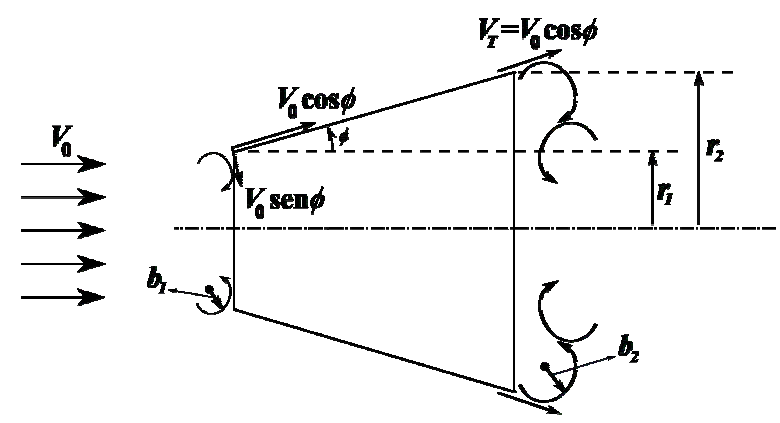

Figure 2. Model of the vortices at the inlet and outlet of the diffuser.

Thus, for calculating the circulation $K_{2}$, it was released the hypothesis that the radius of the vortex $b_{2}$ shown in Fig. 2 is proportional to the difference between the radio at the outlet and the inlet of the diffuser $\left(r_{2}-r_{1}\right)$. Besides, the tangential velocity $V_{T}$ is proportional the product of the undisturbed velocity $V_{0}$ by the cosine of the opening angle $\phi$ of the diffuser. Therefore, the circulation $K_{2}$ is given by: 


$$
K_{2}=\xi_{2} V_{0} \cos \phi\left(r_{2}-r_{1}\right)
$$

where $\xi_{2}$ is a proportionality constant and acts as a correction factor (Eq. 3).

$$
K_{1}=\xi_{1} V_{0} \sin \phi\left(r_{2}-r_{1}\right) \frac{C_{1}}{L+C_{2}}
$$

To determine the circulation $K_{1}$, it was used the same hypothesis, applied to the input of the diffuser, and then $K_{1}$ is given by Eq. 4 .

$$
u(x)=V_{0}+u_{*}(x)
$$

Van Beveren (2008) showed that the velocity profile of the internal diffuser is given by the sum of the velocity induced by the ring vortex and undisturbed flow velocity (Eq. 5).

\section{EXPERIMENTS}

Model validation was performed in an experimental study of airflow around a conical diffuser, in order to obtain the velocity profile along the axial length of the diffuser. Figure 3 illustrates the experimental apparatus. The dimensions of the diffusers are: inlet diameter of $115 \mathrm{~mm}$, outlet diameter of $259 \mathrm{~mm}$ and length of $87 \mathrm{~mm}$. These dimensions were therefore used in the mathematical model proposed in this work.
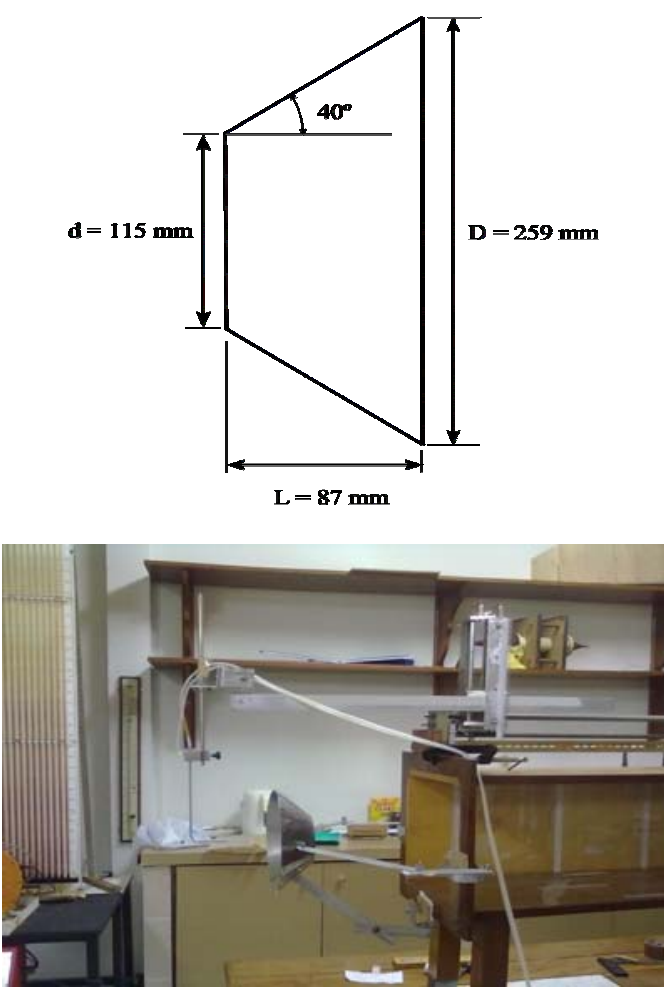

Figure 3. Dimensions of the diffuser and experimental apparatus.
The experiment was conducted in a wind tunnel (controlled by frequency inverter) operating at five different rotations, $400 \mathrm{rpm}, 500 \mathrm{rpm}, 600 \mathrm{rpm}, 700$ $\mathrm{rpm}$ and $800 \mathrm{rpm}$. The measurements were performed with the diffuser positioned externally to the wind tunnel, under the action of a flow in a jet of air. This configuration was chosen due to cross section of the wind tunnel (310 $\mathrm{mm} \times 310 \mathrm{~mm}$ ) to be insufficient to hold the body of the diffuser and avoids wall effects on the flow around the diffuser.

\section{RESULTS AND DISCUSSION}

The velocity values for each point along the longitudinal axis of the diffuser were obtained from a sample of 2000 measurements. Figure 4 shows the velocity distributions calculated by the Eq. (5) compared with values obtained experimentally. The velocity ratio from the furthest region from the diffuser (non-disturbed flow) to its minimum value appears differently from the actual case. From this point of minimum to the maximum point, the velocity distribution shows very good agreement with experimental results, diverging from the point of maximum to the region downstream of the diffuser.

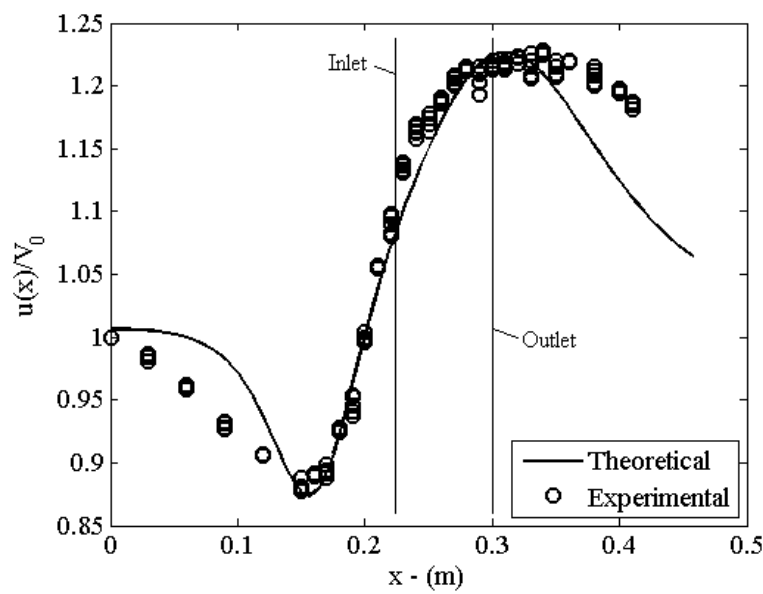

Figure 4. Comparative curve of the theoretical and experimental velocity ratios.

Figure 5 shows the behavior of the velocity profile in the internal region of the diffuser, where it was observed that the results obtained with the proposed model is in agreement. The parameters considered in the simulation were $c_{1}=0.06 \mathrm{~m}, c_{2}=0.0$ $\mathrm{m}, \xi_{1}=0.7$ and $\xi_{2}=1.1$.

\section{CONCLUSIONS}

The velocity distribution obtained with the proposed model presents a good agreement with experimental data. It is noteworthy that the velocity profile matches very well with the experimental data at the internal region of the diffuser. This agreement is important because it is possible to find the best 
position to install the turbine.

Experimental data for other configurations of conical diffusers are currently being obtained to validate and refine the mathematical model proposed. It was also observed that the ratio between length and diameter of the diffuser inlet (aspect ratio) is closely related to the location of a maximum velocity ratio. Therefore, the proposed model has limitations, but it can be applied to DAWTs project, aiming at an improvement in the extraction of energy from the wind, resulting in increased efficiency.

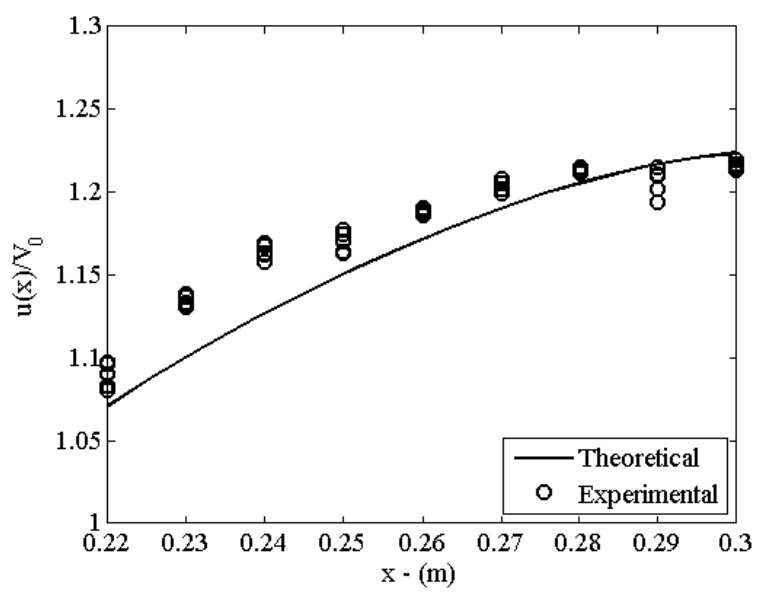

Figure 5. Velocity distribution internally to the diffuser.

\section{ACKNOWLEDGEMENTS}

The authors would like to thank the Eletronorte/Eletrobras Company via Project TUCUNARÉ-Hydrokinetic turbines to exploit the potential remaining in power plants through a doctoral degree scholarship; and $\mathrm{CNPq}$-National Council for Scientific and Technological Development of the Brazil for financially supporting this work through National Institute of Renewable Energy and Energy efficiency in the Amazon-INCT-EREEA.

\section{REFERENCES}

Betz, A., 1926, Wind Energy and its Utilization by Windmills, Vandenhoeck. (in German)

Bussel, G. J. W. V., 1999, An Assessment of the Performance of Diffuser Augmented Wind Turbines (DAWT's), in: 3rd ASME/JSME Joint Fluids Engineering Conference, California, San Francisco.

Foreman, K. M., and Gilbert, B. L., 1979, Technical Development of the Diffuser Augmented Wind Turbine (DAWT) Concept, in: Wind Energy Innovative Systems Conf. Proc., Colorado Springs, Colorado, pp. 121-134.

Hansen, M. O. L, Sorensen, N. N., and Flay, R. G. J., 2000, Effect of Placing a Diffuser Around a wind Turbine, Wind Energy, Vol. 3, pp. 207-213. 